\title{
Analysis of methods for extracting matrix proteins from human kidney stones
}

\begin{abstract}
Five techniques for the extraction of proteins from kidney stones were compared for the purpose of maximizing yield for mass spec analysis. The proteomics of kidney stones may provide insight into the underlying mechanisms of stone formation and why some people are more prone to developing kidney stones than others. Kidney stones contain proteins that may serve as markers for a wide variety of disease states. Mass spectrometry offers the ability to identify and quantify the complete proteome of a kidney stone. To date, there have been only a limited number of research projects aimed at studying the protein composition of kidney stones, possibly due to the difficulty in extracting these proteins while preserving the ability to subject the samples to mass spectrometry. We compared five methods for extracting kidney stone matrix proteins, three from previously published studies and two methods we designed based on previous studies. We were able to establish which methods produced the highest yield of protein for both calcium oxalate and uric acid stones.
\end{abstract}

Volume 4 Issue I - 2017

\author{
Aryeh Stock, Kamlesh Kumar Yadav, Mantu \\ Gupta \\ Department of Urology, Icahn School of Medicine at Mount
} Sinai, USA

Correspondence: Aryeh Stock, Department of Urology, Icahn School of Medicine at Mount Sinai, 1245 Park Ave. 9G New York, NY 10128, USA, Tel 973-5I8-1235, Email stockari@gmail.com

Received: October 21, 2016 | Published: January 5, 2017

Keywords: percutaneous nephrolithotomy, renal stone, ureteral stones, urolithiasis, obstruction

Abbreviations: SMP, stone matrix protein; SLB, stone lysis buffer; DEB, demineralization extraction buffer; LB, loading buffer; $\mathrm{CaOx}$, one calcium oxalate stone; UA, uric acid; SLB, stone lysis buffer; DEB, demineralization extraction buffer; HAS, human serum albumin; MS, mass spectrometry; HA, hydroxyapatite; BR, brushite

\section{Introduction}

Urolithiasis has plagued mankind for millennia and represents a universal health problem. With renal calculi affecting 5-10\% of the European and North American population, it represents a significant burden on the health care system. ${ }^{1}$ The past few decades have seen significant development in the techniques for calculus removal. Today, almost all stones can be removed via minimally invasive procedures. However, the problem of recurrent stone formation remains and efforts at preventing recurrent stone formation have been insufficient. Kidney stone crystal composition is well understood and crystal/mineral composition analysis is a routine part of stone removal. Crystal composition directs clinical decision making. It may determine the method of lithotripsy employed, whether to alkalinize or acidify the patient's urine, or it may indicate an underlying etiology. ${ }^{2-4}$ Understanding the molecular underpinnings of the process of stone formation, both de novo and recurrent, will tremendously aid in not only treatment but also prevention. Towards this end, the proteomics of kidney stones are likely to provide greater insight into the etiologies of calculus formation and may guide treatment of frequent stone formers and patients with certain diseases such as diabetes, obesity, gout, and Crohn's disease known to be associated with kidney stones. ${ }^{4}$ However, kidney stone matrix protein (SMP) composition is poorly established and very little research has been carried out that correlates protein composition with clinical findings.

A number of studies have used mass spectrometry to identify the proteins within kidney stone matrices..$^{5-15}$ These studies have employed a range of techniques to extract proteins from the crystalline structure. Most of these studies have assessed relatively small cohorts of samples and inadequate extraction techniques have necessitated the use of pooled samples. For SMP proteomic studies to be applied to the identification of specific disease states, more productive methods for extracting proteins will need to be established. Single stone analysis is necessary to apply kidney stone proteomics to clinical pathology. Additionally, a standard protocol for the extraction of SMPs will allow for greater reproducibility of results. We sought to compare different SMP extraction methods for their efficiency and reproducibility.

\section{Methods}

We compared five methods (three already published) for extracting proteins from kidney stone matrices (Figure 1). Significant stone sample amounts from individual patients were equally divided and subjected to the 5 different methodologies: 1) Stone Lysis Buffer (SLB) $(0.06 \mathrm{M}$ Tris $\mathrm{HCl}, 10 \%$ glycerol, $5 \% \beta$-mercaptoethanol, $2 \%$ SDS), 2) Demineralization Extraction Buffer (DEB) (0.2 M EDTA, $1 \% \beta$-mercaptoethanol), 3) 1X Loading Buffer (LB) (4X Laemmli Sample Buffer, Bio-Rad diluted 3:1), 4) SLB+EDTA (50\% SLB, 40\% 0.5M EDTA), and 5) LB+EDTA (25\% 4X LB, 40\% 0.5M EDTA). Methods 4 and 5 were rationally designed from previous studies. All buffers were supplemented with protease inhibitors. Extracts were then submitted to SDS-PAGE and stained with Coomassie blue to visualize extracted proteins.

\section{Stone collection}

Deidentifed renal calculus samples were collected from patients undergoing PCNL at Mount Sinai Medical Center under IRB \#HS: 15-00552. Stone composition was determined by a commercial laboratory (Quest Diagnostics, Madison, NJ). Stones were stored at $-80^{\circ} \mathrm{C}$ to prevent protein degradation.

\section{Protein isolation}

One calcium oxalate stone $(\mathrm{CaOx})$ sample and one uric acid 
(UA) stone sample were used for this study. Stones were mixed with dry ice and ground in a ceramic mortar with a marble pestle. The complete stone sample from an individual patient was ground to ensure homogeneity of the sample. Five buffers were prepared: 1) Stone Lysis Buffer (SLB) (0.06 M Tris $\mathrm{HCl}, 10 \%$ glycerol, 5\% $\beta$-mercaptoethanol, 2\% SDS), 2) Demineralization Extraction Buffer (DEB) (0.2 M EDTA, 1\% $\beta$-mercaptoethanol), 3) 1X Loading Buffer (LB) (4X Laemmli Sample Buffer, Bio-Rad diluted 3:1), 4) SLB+EDTA (50\% SLB, 40\% 0.5M EDTA), and 5) LB+EDTA (25\% $4 \mathrm{X} \mathrm{LB}, 40 \% 0.5 \mathrm{M}$ EDTA). $200 \mathrm{mg}$ of stone powder was added to 300 $\mu 1$ of buffer to yield 10 samples. CaOx samples 1,2 , and 4 , and UA samples 1,2 , and 4 were rotated at $4^{\circ} \mathrm{C}$ overnight. The samples were then centrifuged at $4^{\circ} \mathrm{C}$ at $15,000 \mathrm{rpm}$ for 15 minutes in an Eppendorf refrigerated centrifuge (Eppendorf, San Diego, CA, USA; 5424R) and stored at $-20^{\circ} \mathrm{C}$. $\mathrm{CaOx}$ samples 3 and 5 , and UA sample 3 and 5 were boiled at $100^{\circ} \mathrm{C}$ for 15 minutes, centrifuged at $4^{\circ} \mathrm{C}$ at $15,000 \mathrm{rpm}$ for 15 minutes and stored at $-20^{\circ} \mathrm{C}$.

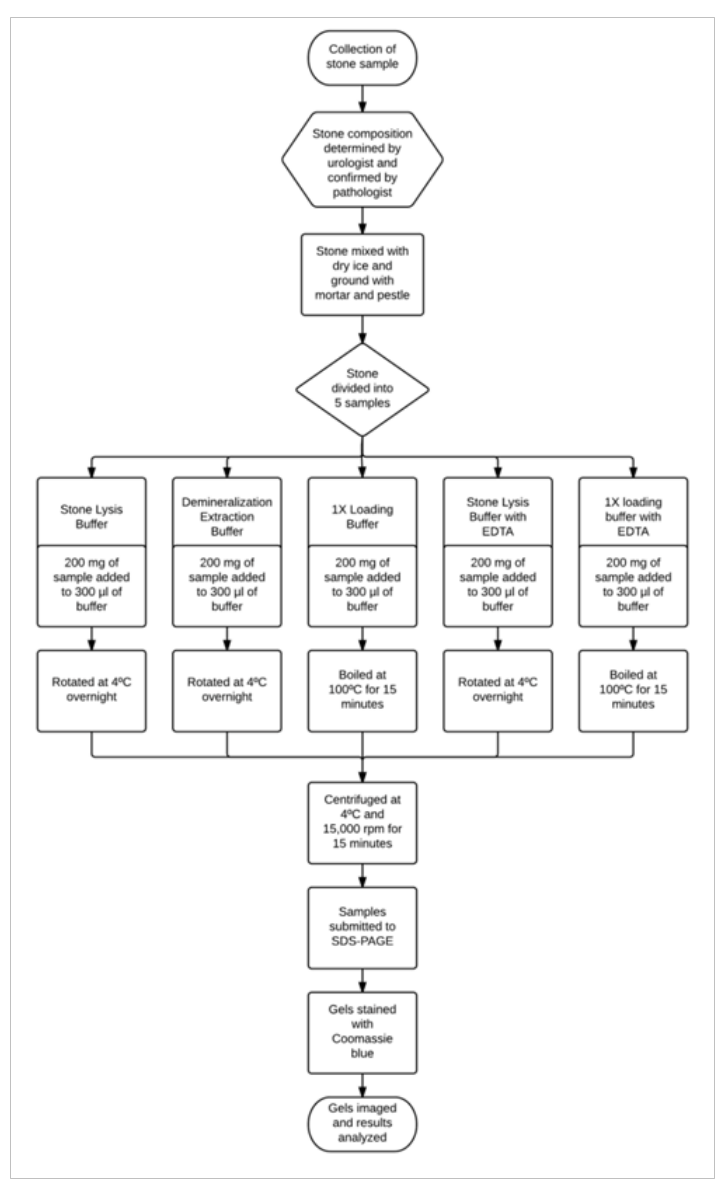

Figure I Stone Extraction Process.

\section{Electrophoretic techniques}

The supernatants of samples 1,2 , and 4 were added 3:1 to $4 \mathrm{X}$ SDS-PAGE sample buffer. All the samples were then separated by electrophoresis in $8 \%$ polyacrylamide gels in the presence of SDS (SDS-PAGE). The gels were then stained with Coomassie brilliant blue R-250 staining solution (Bio-Rad, Hercules, CA, USA; 1610436). The gels were imaged in an Image Quant LAS 4000 (GE, Pittsburgh, PA, USA; 28-9558-10). All reagents were of the highest purity commercially available. Chemicals were from Bio-Rad (Hercules, CA, USA) or Fisher Scientific (Pittsburgh, PA, USA) except glycerol which was from Sigma Aldrich (St. Louis, MO, USA; G5516).

\section{Results}

In general, all of the previously published methods were capable of extracting matrix proteins. However, for calcium oxalate stones, SLB+EDTA (method 4), the method designed by us, provided the best protein extraction (Figure 2). This method also demonstrated the least amount of smearing on the gel, suggesting that it minimized protein degradation. SLB generated a higher yield, but at the cost of significant smearing, indicating a much greater degree of protein degradation. DEB and LB+EDTA did not produce as significant a yield of protein. For uric acid stones, the best protein extraction was produced by SLB (method 1). LB (method 3) provided the overall best protein yield but smeared significantly on SDS-PAGE, likely due to protein degradation (Figure 3). None of the EDTA-containing buffers were able to extract any proteins, presumably due to the nonpresence of divalent cations for EDTA to chelate, thereby reducing the effectiveness of stone solubilization and extraction. Similar to results seen in similar studies, a $67-\mathrm{kDa}$ protein is the most common protein in both the $\mathrm{CaOx}$ and the UA stones. This is most likely human serum albumin as identified previously. ${ }^{7}$ Other bands of approximately $100 \mathrm{kDa}, 60 \mathrm{kDa}$, and $50 \mathrm{kDa}$ can be seen in the $\mathrm{CaOx}$ gel and bands of approximately $100 \mathrm{kDa}, 55 \mathrm{kDa}, 50 \mathrm{kDa}, 35 \mathrm{kDa}$, and $22 \mathrm{kDa}$ can be seen in the UA gel.

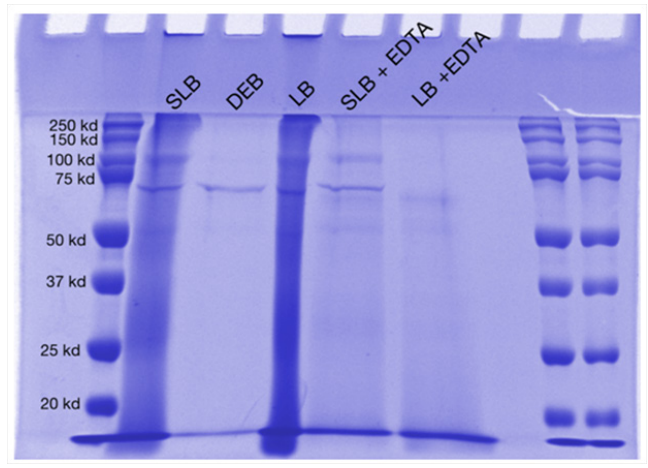

Figure 2 SDS-PAGE gel stained with Coomassie blue. Powdered kidney calculi from calcium oxalate stones were suspended in Stone Lysis Buffer (40 $\mu \mathrm{l}, \mathrm{SLB})$, Demineralization Extraction Buffer (40 $\mu \mathrm{l}, \mathrm{DEB})$, Loading Buffer (40 $\mu \mathrm{l}, \mathrm{LB})$, Stone Lysis Buffer with EDTA (40 $\mu \mathrm{l}, \mathrm{SLB}+\mathrm{EDTA})$, and Loading Buffer with EDTA (40 $\mu \mathrm{l}, \mathrm{LB}+\mathrm{EDTA})$. Molecular weight standard values are indicated to the left the gel.

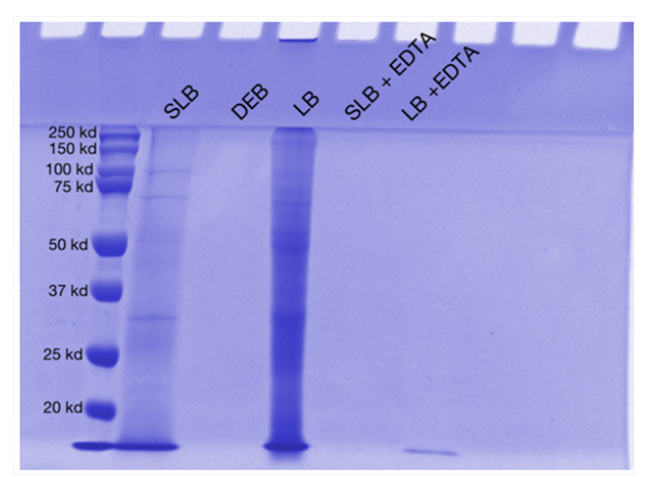

Figure 3 SDS-PAGE gel stained with Coomassie blue. Powdered kidney calculi from uric acid stones were suspended in Stone Lysis Buffer $(40 \mu \mathrm{l}, \mathrm{SLB})$, Demineralization Extraction Buffer $(40 \mu \mathrm{l}, \mathrm{DEB})$, Loading Buffer $(40 \mu \mathrm{l}, \mathrm{LB})$, Stone Lysis Buffer with EDTA (40 $\mu$ l, SLB+EDTA), and Loading Buffer with EDTA (40 $\mu \mathrm{l}, \mathrm{LB}+\mathrm{EDTA})$. Molecular weight standard values are indicated to the left the gel. 


\section{Discussion}

Recent efforts at identifying kidney stone matrix protein composition are few and far in between, and existing studies do not correlate the protein composition with clinical findings. In 1992 Dussol et al. ${ }^{7}$ analyzed matrix protein composition by extracting proteins and submitting them to SDS-PAGE and analyzing by immunoblotting with antibodies to 13 common urinary proteins. They analyzed stones of 5 different crystal compositions and identified 9 proteins common to all types of stone: human serum albumin (HSA), $\alpha 1$-acid glycoprotein, a1-microglobulin, immunoglobulins, apolipoprotein A1, transferrin, $\alpha 1$-antitrypsin, retinol-binding protein, and renal lithostathine. Dussol et al. ${ }^{7}$ also found that HSA induced the binding of matrix proteins to calcium oxalate monohydrate crystals in a urine-like solution. Mass spectrometry (MS) analysis has enabled the analysis of complete proteomes. Proteins are digested enzymatically by a protease into smaller peptides.

The mass spectrometer identifies sequences of proteins. Unique sequences are compared to a database of known peptide sequences and the probability of a given protein being present can be calculated. With MS, we have the potential to map complete proteomes of kidney stone matrix proteins In 2008, Canales et al. ${ }^{16}$ used reversed-phase, high-performance liquid chromatography followed by MS/MS (tandem MS) spectrometry compared against the National Center for Biotechnology Information's human-subset, nonredundant protein database to identify 68 distinct proteins. From 7 calcium oxalate monohydrate stones, 23 inflammatory proteins were identified (13 of which were found in more than one stone). Merchant et al. ${ }^{13}$ used MS-MALDI compared against a Swiss Prot human protein database and ESI-MS compared against a translated human genome database. They identified 158 proteins in stones collected from 5 patients. Their findings support the hypothesis that inflammatory cells play an integral role in the production of stone matrix proteins. Additionally they found significant amounts of myeloperoxidase and osteopontin which supports the hypothesis that proteolytic activity plays a role in stone formation. ${ }^{13}$

In 2010 Thurgood et al. ${ }^{17}$ sought to distinguish between intracrystalline and extracrystalline proteins. They induced crystal precipitation in healthy urine samples by adding aqueous sodium oxalate. Crystals were washed with water and $\mathrm{NaOH}$. Proteins identified after washing with $\mathrm{NaOH}$ were assumed to represent intracrystalline proteins. Thurgood et al. ${ }^{17}$ were able to identify only 14 proteins present in healthy urine that were consistently incorporated into calcium oxalate monohydrate crystals and 34 proteins that were consistently incorporated into dihydrate crystals. Thurgood et al. ${ }^{12}$ also precipitated hydroxyapatite (HA), uric acid (UA), and brushite (BR) crystals but were only able to produce all three crystals from a single urine sample on two occasions. They identified 13 proteins common to both HA samples out of a total of 36,3 proteins common to both BR samples out of a total of 65 , and only 1 protein in common to both UA samples out of a total of 7. Thurgood et al.'s ${ }^{12}$ methods provide a potential framework for future studies on kidney stone matrix proteomes.

Between 2010 and today, a number of papers have been published that add to the total number of proteins known to have been identified in kidney stones matrices. ${ }^{5,6,8,9,14,18,19}$ These papers point to the large proportion of inflammatory proteins found within kidney stone matrices. They also highlight the diversity in kidney stone matrix protein composition between individuals. In 2016 Witzmann et al. ${ }^{15}$ reported identifying a staggering 1,059 unique proteins from two $\mathrm{CaOx}$ stone samples. They utilized a novel extraction method involving $8 \mathrm{M}$ urea and DL-Dithiothreitol, a $190 \mathrm{~min}$ liquid chromatography gradient, and a more advanced mass spectrometer to maximize protein yields beyond those of previous studies. A standardized process for extracting proteins from kidney stones will facilitate the application of proteomics to clinical pathology. We observed that SLB provided the greatest overall yield of protein from calcium oxalate stones, (Figure 2). However, this is accompanied by smearing of the lane, likely due to fragmented proteins. The addition of EDTA significantly improves the quality of the protein extracted. The band intensities are similar for SLB and SLB+EDTA, however, the latter is accompanied by far less smearing on the gel. This is likely due to EDTA's tendency to chelate calcium ions, effectively dissolving away the stone releasing bound proteins thereby reducing aberration and protein degradation. Thus, for stones known to be primarily $\mathrm{CaOx}$, the SLB+EDTA extraction method proved to be the most effective, providing a good balance between protein extraction and protein degradation.

For stones known not to contain calcium or stones of unknown composition, SLB provided the cleanest extraction of proteins with sharp, well-defined bands (Figure 3). Not surprisingly, EDTAcontaining buffers were ineffective at extracting proteins from noncalcium containing stones. LB, however, provided the overall greatest protein yield. Therefore, for studies in which protein degradation is of less significance (e.g. those utilizing mass spectrometry) the LB extraction method may prove the most effective. To keep the comparison accurate, the addition of protease inhibitors to the extraction buffers was employed. This allowed us to run the extraction for much longer than would otherwise be possible, allowing for a far greater overall yield of protein.

One aspect of the extraction process that proved to be particularly challenging was the pulverization of the stones. Our initial attempt to sonicate stone samples under liquid nitrogen, to maintain as low a state of entropy as possible while pulverizing our samples and avoiding the use of a solvent, provided inconsistent results as it was challenging to control the temperature of the buffer. It also did not produce uniform stone gravel. Subsequently, using a mortar filled with liquid nitrogen and grinding the stones with a pestle enabled us to generate a fairly uniform powder; however, the liquid nitrogen caused the stone to form slurry that proved difficult to measure accurately, leading to inconsistent results. Finally, by mixing dry ice with the stone samples and then crushing the mixture in a mortar and pestle, the samples remained cold without forming a thick slurry, making it easier to transfer the ground stone-dry ice mixture out of the mortar and allowing the dry ice to sublimate off the sample, leaving behind a dry loose powder.

We have identified a number of factors that can be improved upon in further studies. Mass spectroscopy is an imperfect tool for the identification of proteins. While the cost is dropping rapidly, the expense of processing the number of samples needed for a pathophysiology-oriented study is prohibitive. Most prior studies were able to identify a large number of proteins but unable to distinguish "bystander" proteins present in the urine or coating the outer surface of the kidney stone from significant proteins incorporated into the kidney stone itself. Quantitative mass spectroscopy, an emerging technology, will allow for the quantification necessary to establish which proteins are fundamental to the structure of kidney stones, and which are bystanders. Finally, prior studies utilized inefficient methods of extraction that produced miniscule yields of protein, necessitating 
use of pooled samples. A clinically driven study requires extraction methods that are inexpensive, rapid, and accurate while maximizing the yield of protein. We focused our study on pursuing these goals. Developing improvements in the protein extraction from kidney stones will serve as a springboard for more focused and treatmentdirected research on nephrolithiasis.

Much progress has been made in defining the proteomics of kidney stones. The process of identifying proteins has advanced from Western blotting (reliant upon antibodies targeted to specific antigens) to mass spectrometry capable of sequencing protein fragments for submission to peptide mass fingerprinting for comparison against a database of known protein fragment masses. Future studies will likely benefit from emerging technologies that allow for protein quantization or rapid de novo protein sequencing. We believe that the next step in stone matrix protein analysis is to identify which proteins are critical to stone formation. This would guide our understanding of the principles that drive urolithiasis. We also feel that it would be of benefit to search for correlations between stone protein composition and specific disease states. Are there matrix proteins associated with certain diseases? Can kidney stones serve as markers or even timelines of disease progression?

\section{Conclusion}

For stones known to be composed primarily of calcium oxalate, the SLB+EDTA extraction method proved to be the most effective. It provides a good balance between protein extraction and degradation. For stones known not to contain calcium or stones of unknown composition, the SLB extraction method provides the best overall extraction of proteins. These results will be invaluable for correlating the protein composition of the stones in a particular individual with his or her clinical findings, bringing us one step closer to precision medicine.

\section{Acknowledgement}

Thank you to Dr. Ashutosh Tewari for use of his laboratory space and reagents.

\section{Conflicts of interest}

The author declares there is no conflict of interest.

\section{References}

1. Tiselius HG. Epidemiology and medical management of stone disease BJU Int. 2003;91(8):758-767.

2. Bihl G, Meyers A. Recurrent renal stone disease-advances in pathogenesis and clinical management. Lancet. 2001;358(9282):651-656.

3. Coe FL, Parks JH, Asplin JR. The Pathogenesis and Treatment of Kidney Stones. N Engl J Med. 1992;327(16):1141-1152.
4. Coe FL, Evan A, Worcester E. Kidney stone disease. J Clin Invest. 2005;115(10):2598-2608.

5. Aggarwal KP, Tandon S, Naik PK, et al. Novel antilithiatic cationic proteins from human calcium oxalate renal stone matrix identified by MALDI-TOFMS endowed with cytoprotective potential: An insight into the molecular mechanism of urolithiasis. Clin Chim Acta. 2013;415:181-190.

6. Aggarwal KP, Tandon S, Naik PK, et al. Peeping into Human Renal Calcium Oxalate Stone Matrix: Characterization of Novel Proteins Involved in the Intricate Mechanism of Urolithiasis. PLoS ONE. 2013;8(7):e69916.

7. Dussol B, Geider S, Lilova A, et al. Analysis of the soluble organic matrix of five morphologically different kidney stones. Evidence for a specific role of albumin in the constitution of the stone protein matrix. Urol Res. 1995;(231):45-51.

8. Okumura N, Tsujihata M, Momohara C, et al. Diversity in Protein Profiles of Individual Calcium Oxalate Kidney Stones. PLoS ONE. 2013;8(7):e68624.

9. Boonla C, Tosukhowong P, Spittau B, et al. Inflammatory and fibrotic proteins proteomically identified as key protein constituents in urine and stone matrix of patients with kidney calculi. Clin Chim Acta. 2014;429:8189.

10. Chen WC, Lai CC, Tsai Y, et al. Mass spectroscopic characteristics of low molecular weight proteins extracted from calcium oxalate stones: preliminary study. J Clin Lab Anal. 2018;22(1):77-85.

11. Liu JD, Liu JJ, Yuan JH, et al. Proteome of melamine urinary bladder stones and implication for stone formation. Toxicol Lett. 2012;212(3):307-314.

12. Thurgood LA, Ryall RL. Proteomic Analysis of Proteins Selectively Associated with Hydroxyapatite, Brushite, and Uric Acid Crystals Precipitated from Human Urine. J Proteome Res. 2010;9(10):5402-5412.

13. Merchant ML, Cummins TD, Wilkey DW, et al. Proteomic analysis of renal calculi indicates an important role for inflammatory processes in calcium stone formation. Am J Physiol Ren Physiol. 2008;295(4):F1254-F1258.

14. Jou YC, Fang CY, Chen SY, et al.Proteomic Study of Renal Uric Acid Stone. Urology. 2012;80(2):260-266.

15. Witzmann FA, Evan AP, Coe FL, et al. Label-free proteomic methodology for the analysis of human kidney stone matrix composition. Proteome Sci. 2016;14: 4 .

16. Canales BK, Anderson L, Higgins L, et al. Second Prize: Comprehensive Proteomic Analysis of Human Calcium Oxalate Monohydrate Kidney Stone Matrix. J Endourol. 2008;22(6):1161-1168.

17. Thurgood LA, Wang T, Chataway TK, et al. Comparison of the Specific Incorporation of Intracrystalline Proteins into Urinary Calcium Oxalate Monohydrate and Dihydrate Crystals. J Proteome Res. 2010;9(9):47454757.

18. Canales BK, Anderson L, Higgins L, et al. Proteome of Human Calcium Kidney Stones. Urology. 2010;76(4):1017.

19. Aggarwal KP, Tandon S, Singh SK, et al. 2D map of proteins from human renal stone matrix and evaluation of their effect on oxalate induced renal tubular epithelial cell injury. Int Braz J Urol. 2013;39(1):128-136. 